\title{
On the Nature of Suppes-Sen Maximal Paths in an Aggregative Growth Model*
}

\author{
Ram Sewak Dubey ${ }^{\dagger}$ and Tapan Mitra ${ }^{\ddagger}$
}

\begin{abstract}
This paper investigates the nature of paths in the standard neoclassical aggregative model of economic growth that are maximal according to the Suppes-Sen grading principle. This is accomplished by relating such paths to paths which are utilitarian maximal when an increasing (but not necessarily concave) utility function evaluates each period's consumption. Dynamic properties of Suppes-Sen maximal paths, which lie entirely above or entirely below the golden-rule, are analyzed. An example is presented in which an explicit form of a consumption function is described, which generates only Suppes-Sen maximal paths. This consumption function is shown to generate consumption cycles, and violate the Pigou-Dalton transfer principle.

Journal of Economic Literature Classification Numbers: D60, D70, D90.

Keywords and Phrases: Suppes-Sen Grading Principle, Utilitarian Maximality, Aggregative Growth Model, Golden Rule, Non-concave utility function, Consumption Cycle, Pigou-Dalton Transfer Principle.
\end{abstract}

\footnotetext{
${ }^{*}$ We would like to thank an Editor and two referees of this journal for their comments and suggestions on the earlier versions of this paper.

†Department of Economics, Colgate University, Hamilton, NY 13346; E-mail: rdubey@ colgate.edu

$\ddagger$ Department of Economics, Cornell University, Ithaca, NY 14853; E-mail: tm19@ cornell.edu
} 


\section{Introduction}

In ranking social states which are specified by infinite utility streams, it is customary to use a social welfare quasi-ordering (SWQ), a reflexive and transitive binary relation on the social states, satisfying two widely accepted guiding principles. The equal treatment of all generations, proposed by Ramsey (1928), is formalized in the Anonymity Axiom. The positive sensitivity of the social preference structure to the well-being of each generation is reflected in the Pareto Axiom. The Suppes-Sen grading principle ${ }^{1}$ in the context of infinite utility streams can be characterized as the least restrictive SWQ satisfying the Pareto and Anonymity axioms; see d'Aspremont (1985) and Asheim, Buchholz and Tungodden (2001) for this characterization result.

In the context of growth models, where the feasible infinite consumption sequences are determined by the technological specifications and availability of resources of an economy, it would be useful in making intertemporal social choices to determine the nature of feasible growth paths that are maximal according to the Suppes-Sen grading principle ${ }^{2}$.

In the standard aggregative model of economic growth, which allows for a golden-rule steady-state, paths which are intertemporally efficient (in terms of their consumption streams) have been characterized in a classic paper by Cass (1972). When an increasing and strictly concave felicity function evaluates the period consumption, paths which are (catching-up) optimal (in terms of these felicities) have been characterized by Koopmans (1967) and Gale (1967). These provide (respectively) the characterization of paths which are maximal according to the efficiency quasi-ordering, and the catching-up quasi-ordering ${ }^{3}$.

Paths which are maximal according to the grading principle fall in between the two classes mentioned above. Any path which is maximal according to the grading principle is also maximal according to the efficiency quasi-ordering. And, any path which is maximal according to the catching-up quasi-ordering is also maximal according to the grading principle. Our first objective in this paper is to try to describe the nature of paths which are maximal according to the grading principle (which we call Suppes-Sen maximal paths).

In a recent contribution to intertemporal social choice theory, Asheim et al (2010) have investigated the nature of infinite-horizon choice functions in an aggregative productive model of economic growth. An interesting result obtained by them in this framework is that for the class of efficient paths, the choices according to the Suppes-Sen grading principle which embodies the notion of procedural equity coincide with the choices according to the Pigou-Dalton transfer principle which embodies the notion of consequentialist equity, and that both notions lead to the choice of paths which are monotone non-decreasing in consumption over time. A second objective of this paper is to investigate the robustness of this finding by examining the equivalence result in the standard aggregative model of economic growth.

While the two objectives stated above provide the primary motivation for our paper, our research can also be viewed as suggesting a useful approach to the study of Suppes-Sen maximal paths. Permutations involved in the definition of the grading principle make the study of Suppes-Sen maximal paths technically challenging. Our approach shows how to directly exploit the permutations appearing in the definition of the grading principle to derive necessary conditions for a path to be Suppes-Sen maximal. These necessary conditions in turn can be used to address the first objective of our study.

\footnotetext{
${ }^{1}$ The grading principle is due to Suppes (1966). For a comprehensive analysis of it, see Sen (1971). Svensson (1980) provides a formal definition of the Suppes-Sen grading principle in the context of infinite utility streams.

${ }^{2}$ For the purpose of this paper, we will interpret the grading principle as providing certain comparisons between consumption streams, rather than welfare streams, with welfare being derived from consumption, using a period welfare function. Since this is the interpretation used in the recent paper by Asheim et al (2010), this will also facilitate comparison of our work with theirs.

${ }^{3}$ Basu and Mitra (2007) have proposed a new quasi-order, which can be characterized by the Anonymity and Pareto axioms, plus the axiom of unit comparability. It is a less restrictive quasi-order than the catching-up quasi-ordering. However, in the context of the standard aggregative growth model, they have shown that paths which are maximal according to the two quasi-orders coincide.
} 
In order to establish that a path, satisfying some verifiable properties, is Suppes-Sen maximal, we show how to indirectly exploit the permutations appearing in the definition of the grading principle. A key observation $^{4}$ that we use (closely related to a characterization result due to Hardy, Littlewood and Polya (1952)) is that if a path is maximal for the quasi-ordering associated with the notion of weak-maximality of Brock (1970), with an increasing felicity function evaluating the period consumption ${ }^{5}$, then it is also Suppes-Sen maximal ${ }^{6}$. This observation allows us to bring our study under the rubric of "optimal economic growth" and provides us with a convenient sufficient condition for identifying Suppes-Sen maximal paths. Since concavity of the felicity function plays no role in the key observation, a serious study of Suppes-Sen maximal paths provides a clear-cut motivation for studying optimal economic growth without the concavity restriction on the felicity function.

Using the necessary conditions for a path to be Suppes-Sen maximal (mentioned above), we are able to address the first objective of the paper as follows. We establish that if the capital stock sequence of a Suppes-Sen maximal path is always "well below" the golden-rule capital stock ${ }^{7}$, then the capital stock sequence is monotonically non-decreasing over time, and therefore convergent. Because of the well-known Phelps-Koopmans result, the capital stock sequence along any Suppes-Sen maximal path cannot be above and bounded away from the golden-rule capital stock; so our result, when the capital stock sequence along any Suppes-Sen maximal path is always above the golden-rule capital stock, is necessarily asymmetric to the result described above. We show that in such cases, the capital stock sequence either (a) converges to the golden-rule capital stock, or (b) it generates "persistent fluctuations 8 ".

It is important at this stage to point out that much of the theory of optimal economic growth is based on the assumption that an increasing and concave (indeed strictly concave) felicity function evaluates the period consumption. And, under this assumption, it is well-known that any path, starting with a capital stock above the golden-rule capital stock, which is maximal in terms of the catching-up quasi-order, converges to the golden-rule. Since such paths are necessarily Suppes-Sen maximal, the scenario (a) should come as no surprise. However, scenario (b) would clearly distinguish the maximal paths under the Suppes-Sen quasi-order from those under the catching-up quasi-order, and therefore it is important to establish that it can actually occur. It is precisely at this point that we need to use the sufficient condition for identifying Suppes-Sen paths.

The intuition for scenario (b) to occur can be described as follows. As soon as one allows the class of felicity functions to exhibit non-concavities, a primary reason for preferring to smooth out consumption over time disappears, and optimal growth, even in the case where future felicities are not discounted ${ }^{9}$, can be compatible with cyclical behavior of consumption over time. This, in turn, suggests strongly that SuppesSen maximal paths need not be monotonic in our framework, and may in fact exhibit consumption and capital stock cycles. This intuition is confirmed by establishing rigorously in an example ${ }^{10}$ of the aggregative neoclassical model that there is a Suppes-Sen maximal path which cycles between the golden-rule capital stock and a higher capital stock.

The theory of optimal economic growth, under the assumption that an increasing and strictly concave felicity function evaluates the period consumption, tells us that the golden-rule path is catching-up maximal.

\footnotetext{
${ }^{4}$ This observation is straightforward for increasing felicity functions. But, for reasons that will be clear in Section 4 , we define the class of felicity functions somewhat more broadly, and therefore need to establish this result as a key tool (see Proposition 1 in Section 3).

${ }^{5}$ If the felicity function is denoted by $w$, we call such a path w-maximal in this paper.

${ }^{6} \mathrm{We}$ call such a path $\mathrm{S}$-maximal in this paper.

${ }^{7}$ Precisely, the output producible from the capital stock is below the golden-rule capital stock.

${ }^{8}$ Precisely, it is bounded away from the golden-rule stock for a subsequence of time periods, and converges to the golden-rule stock for another subsequence of time periods.

${ }^{9}$ The fact that discounting future felicities can lead to regular cycles, in models of optimal growth with a strictly concave felicity function, is of course very well understood by this time.

${ }^{10} \mathrm{We}$ use a technology specified by a piecewise linear production function, with the golden-rule at the kink.
} 
It is therefore also Suppes-Sen maximal. This fact, together with the example described in the previous paragraph, indicate that there can be multiple Suppes-Sen maximal paths starting from the same initial capital stock. In their study, Asheim et al (2010) focus on paths which are generated by a consumption function. A Suppes-Sen consumption function would then generate a unique path from each initial stock, and this path would be Suppes-Sen maximal. Their equivalence result establishes (in an aggregative productive model of economic growth) that the class of Suppes-Sen consumption functions coincides with the class of consumption functions, which generate only paths that are efficient and maximal according to the PigouDalton transfer principle.

We address the second objective of this paper by presenting an example of the aggregative neoclassical model, in which we can explicitly solve for a Suppes-Sen consumption function. This consumption function is obtained by appealing to the sufficient condition for identifying Suppes-Sen maximal paths. It is a considerably more subtle example to construct, since one has to show that, using this consumption function, one generates Suppes-Sen maximal paths starting from every initial capital stock. We verify that such a consumption function generates cyclic paths that cannot be maximal according to the Pigou-Dalton transfer principle.

The paper is organized as follows. The framework is described in Section 2. The link between a SuppesSen maximal path and a path which is maximal for the quasi-ordering associated with the notion of weakmaximality of Brock (1970) is established in Section 3. The dynamic properties of Suppes-Sen maximal paths (with capital stocks always above or always below the golden-rule capital stock) are described in Section 4. In Section 5, we explicitly solve for a consumption function which generates only Suppes-Sen maximal paths, and show that such paths (i) can be cyclical, and (ii) violate the Pigou-Dalton transfer principle. Section 6 contains a few concluding remarks, including an open question. Section 7 collects together the proofs of all the results.

\section{The Framework}

\subsection{Notation}

Let $\mathbb{N}$ denote, as usual, the set of natural numbers $\{1,2,3, \ldots\}$, let $\mathbb{N}_{0}$ denote the set $\{0,1,2,3, \ldots\}$, and let $\mathbb{R}$ denote the set of real numbers. For $c, c^{\prime} \in \mathbb{R}^{\mathbb{N}}$, we write $c^{\prime} \geq c$ if $c^{\prime}(t) \geq c(t)$ for all $t \in \mathbb{N}, c^{\prime}>c$ if $c^{\prime} \geq c$, and $c^{\prime} \neq c$, and $c^{\prime}>>c$ if $c^{\prime}(t)>c(t)$ for all $t \in \mathbb{N}$.

\subsection{Consumption Possibilities in an Aggregative Model}

We begin by describing the aggregative neoclassical model of economic growth ${ }^{11}$. The framework is described by a function $f$, where $f$ represents the production function. The production function, $f: \mathbb{R}_{+} \rightarrow \mathbb{R}_{+}$ will be supposed to satisfy the following assumptions:

(F.1) $f(0)=0, f$ is increasing, concave and continuous on $\mathbb{R}_{+}$.

(F.2) There is $K \in(0, \infty)$, such that $f(x)>x$ for all $x \in(0, K)$, and $f(x)<x$ for all $x>K$.

We refer to $K$ as the maximum sustainable stock. It can be shown that there exists $z \in(0, K)$ such that:

$$
f(z)-z \geq f(x)-x \text { for all } x \geq 0 .
$$

\footnotetext{
${ }^{11}$ Our version is more general than the one used in Basu and Mitra (2007), since we do not use any smoothness assumption on the production function.
} 
We will assume that this $z$ is unique ${ }^{12}$. An important implication of this is:

$$
\left.\begin{array}{l}
\text { (i) }[f(x)-x] \uparrow \text { as } x \uparrow \text { for } x \in[0, z] \\
\text { (ii) }[f(x)-x] \downarrow \text { as } x \uparrow \text { for } x \in[z, K]
\end{array}\right\}
$$

We refer to $z$ as the golden rule stock and $g=f(z)-z$ as the golden-rule consumption.

A feasible path from $\kappa \geq 0$ is a sequence of capital stocks $\{k(t)\}$ satisfying:

$$
k(0)=\kappa, 0 \leq k(t+1) \leq f(k(t)) \text { for } t \in \mathbb{N}_{0} .
$$

Associated with the feasible path $\{k(t)\}$ from $\kappa$ is a consumption sequence $\{c(t+1)\}$, defined by:

$$
c(t+1)=f(k(t))-k(t+1) \text { for } t \in \mathbb{N}_{0} .
$$

Sometimes, to emphasize this association, we will denote a feasible path by $\{k(t), c(t+1)\}$.

The infinite horizon consumption possibility set from the initial stock $\kappa$ is defined as:

$C(\kappa)=\{(c(1), c(2), \ldots):\{c(t+1)\}$ is a consumption sequence associated with a feasible path $\{k(t)\}$ from $\kappa\}$.

It is easy to show that for every feasible path $\{k(t)\}$ from $\kappa \geq 0$, we have:

$$
k(t) \leq M(\kappa) \text { for } t \in \mathbb{N}_{0} ; c(t+1) \leq M(\kappa) \text { for } t \in \mathbb{N}_{0}
$$

where $\max \{K, \kappa\} \equiv M(\kappa)$. We will confine our discussion to feasible paths starting from initial stocks $\kappa \in[0, K] \equiv Y$. Then, since $M(\kappa)=K$, it follows that for every feasible path $\{k(t)\}$ from $k \in[0, K]$, we have:

$$
k(t) \leq K \text { for } t \in \mathbb{N}_{0} ; c(t+1) \leq K \text { for } t \in \mathbb{N}_{0} .
$$

A feasible path $\left\{k^{\prime}(t)\right\}$ from $\kappa$ dominates a feasible path $\{k(t)\}$ from $\kappa$, if:

$$
c^{\prime}(t) \geq c(t) \text { for all } t \in \mathbb{N}
$$

with strict inequality holding in for at least some $t \in \mathbb{N}$. A feasible path $\{k(t)\}$ from $\kappa$ is called inefficient if there is a feasible path $\left\{k^{\prime}(t)\right\}$ from $\kappa$ which dominates it. It is efficient if it is not inefficient.

\subsection{The Suppes-Sen Grading Principle}

A social welfare relation (SWR) is a binary relation on $C(\kappa)$. A social welfare quasi-ordering (SWQ) is a SWR, $\succsim$, on $C(\kappa)$, which is reflexive and transitive. We associate with $\succsim$ its symmetric and asymmetric components in the usual way. Thus, we write $x \sim y$ when $x \succsim y$ and $y \succsim x$ both hold; and, we write $x \succ y$ when $x \succsim y$ holds, but $y \succsim x$ does not hold. A SWR $\succsim_{A}$ is a subrelation to a SWR $\succsim_{B}$ if (a) $x, y \in C(\kappa)$ and $x \succsim_{A} y$ implies $x \succsim_{B} y$; and (b) $x, y \in X$ and $x \succ_{A} y$ implies $x \succ_{B} y$.

A permutation $\pi$ is a one-to-one map from $\mathbb{N}$ onto $\mathbb{N}$. Any $c \in C(\kappa)$ can be viewed as a map from $\mathbb{N}$ to $Y$, associating with each $t \in \mathbb{N}$ the element $c(t) \in Y$. The composite map $c \circ \pi$ is then a map from $\mathbb{N}$ to $Y$, associating with each $t \in \mathbb{N}$ an element $\pi(t)$ through the map $\pi$, and then associating the element $c(\pi(t)) \in Y$ through the map $c$. Thus, if $c$ is written as the sequence $(c(1), c(2), \ldots) \in C(\kappa)$, then $c \circ \pi$ is written as the sequence $(c(\pi(1)), c(\pi(2)), \ldots) \in C(\kappa)$. A finite permutation $\pi$ is a permutation, such that there is some $T \in \mathbb{N}$, with $\pi(t)=t$ for all $t>T$. The set of all finite permutations is denoted by $\mathcal{F}$.

\footnotetext{
${ }^{12}$ Alternative sufficient conditions can be placed on $f$ to ensure this uniqueness, but we prefer at this point to simply assume the result itself, to keep the framework more general.
} 
The Suppes-Sen grading principle is a SWQ, denoted by $\succsim_{S}$, defined as follows. Given $c, c^{\prime} \in C(\kappa)$, we write $c^{\prime} \succsim_{S} c$ iff there is a permutation $\pi \in \mathcal{F}$ such that $c^{\prime} \circ \pi \geq c$. A feasible path $\{k(t)\}$ from $\kappa$ is $S$-maximal if its associated consumption sequence $c \equiv\{c(t+1)\}$ is a maximal element of $C(\kappa)$, according to the grading principle. That is, a feasible path $\{k(t)\}$ from $\kappa$ is $S$-maximal if there is no feasible path $\left\{k^{\prime}(t)\right\}$ from $\kappa$, with associated consumption sequence $c^{\prime} \equiv\left\{c^{\prime}(t+1)\right\}$ satisfying $c^{\prime} \succ_{S} c$.

We are interested in characterizing the S-maximal paths from an arbitrary $\kappa \in[0, K]$. These might be considered to represent the choice set of the consumption possibility set $C(\kappa)$, using the preference quasiorder $\succsim s$.

\subsection{A Preliminary Result}

In attempting a characterization of the S-maximal feasible paths, considerable simplification can be obtained by noting a preliminary result, regarding the substitution possibilities of the consumption possibility set. Specifically, given a feasible path $\{k(t)\}$ with positive consumption in some period $T$, it is possible to construct another feasible path which has a bit less consumption in period $T$, but more consumption in all other periods, compared to the consumption sequence on the path $\{k(t)\}$.

Lemma 1 Let $\{k(t)\}$ be a feasible path from $\kappa \in[0, K]$, with associated consumption sequence $\{c(t+1)\}$. Let $T \in \mathbb{N}$, and let $0 \leq \gamma<c(T)$. Then, there is a feasible path $\left\{k^{\prime}(t)\right\}$ from $\kappa$, with associated consumption sequence $\left\{c^{\prime}(t+1)\right\}$, such that $c^{\prime}(T)>\gamma$, and $c^{\prime}(t+1)>c(t+1)$ for all $t \neq T-1$.

The Lemma yields the useful corollary that if a feasible path $\{k(t)\}$ is not S-maximal, then there is a feasible path $\left\{k^{\prime}(t)\right\}$ and a finite permutation such that the permuted consumption sequence on $\left\{k^{\prime}(t)\right\}$ exceeds the consumption sequence on $\{k(t)\}$ in all periods.

Corollary 1 Let $c \in C(\kappa)$, and suppose $c$ is not $S$-maximal. Then, there is $\bar{c} \in C(\kappa)$ and $\pi \in \mathcal{F}$, such that:

$$
\bar{c}(\pi(t))>c(t) \text { for all } t \geq 1 \text {. }
$$

\section{The Grading Principle and Utilitarianism}

Permutations involved in the definition of the grading principle make the study of S-maximal paths technically difficult. It becomes cumbersome to look for permutations under which two consumption streams become comparable according to the quasi-ordering.

This difficulty can be circumvented by relating the grading principle to a utilitarian relation (for a class of felicity functions), so that consumption streams which are maximal according to the utilitarian relation yield consumption streams which are S-maximal. Since the utilitarian relation has been studied extensively in ranking consumption streams in growth models, establishing the connection has the potential of making familiar techniques (which, incidentally, do not involve permutations) available to us for the study of S-maximal paths. This is the approach developed in this section, and applied in the next two sections, demonstrating that it is indeed a fruitful detour.

The result connecting the grading principle and utilitarianism (in comparing finite streams) is directly obtained from a classic characterization reported by Hardy, Littlewood and Polya (1952), in their definitive study on mathematical inequalities.

Lemma 2 (Hardy, Littlewood and Polya) Let $(x(1), \ldots, x(n)),\left(x^{\prime}(1), \ldots, x^{\prime}(n)\right)$ be vectors in $\mathbb{R}^{n}$.

(i) If, for a permutation $\pi$ of the integers $\{1, \ldots, n\}$, we have $\left(x^{\prime}(\pi(1)), \ldots, x^{\prime}(\pi(n))\right) \geq(x(1), \ldots, x(n))$, then for every increasing and continuous function $g: \mathbb{R} \rightarrow \mathbb{R}$,

$$
\sum_{i=1}^{n} g\left(x^{\prime}(i)\right) \geq \sum_{i=1}^{n} g(x(i))
$$


(ii) If, for every increasing and continuous function $g: \mathbb{R} \rightarrow \mathbb{R}$,

$$
\sum_{i=1}^{n} g\left(x^{\prime}(i)\right) \geq \sum_{i=1}^{n} g(x(i))
$$

then there is a permutation $\pi$ of the integers $\{1, \ldots, n\}$, such that $\left(x^{\prime}(\pi(1)), \ldots, x^{\prime}(\pi(n))\right) \geq(x(1), \ldots, x(n))$.

In contrast to Lemma 2, we will be concerned with comparison of infinite streams. The grading principle still involves permutation of a finite number of elements of the stream (although this finite number is not fixed in advance) and therefore does not create additional problems. However, one will have to choose the utilitarian criterion more carefully, since the sums in Lemma 2 will typically not be well-defined for infinite streams.

Another difference is that we will want the utilitarian comparisons for a somewhat broader class of felicity functions than are allowed in Lemma 2 (for reasons which can best be appreciated when the theory is applied in Sections 4 and 5). We take up each of these issues in the following subsections.

\subsection{A Class of Felicity Functions}

Let us denote by $W_{N D}$ the class of non-decreasing functions from $\mathbb{R}_{+}$to $\mathbb{R}$. We define the subclass $W$ of $W_{N D}$ by:

$$
W=\left\{w \in W_{N D}: \text { there exists } \bar{c} \in \mathbb{R}_{+}, \text {such that } w(c)>w(\bar{c}) \text { for all } c>\bar{c}\right\} .
$$

Clearly $W$ includes the class of all increasing functions from $\mathbb{R}_{+}$to $\mathbb{R}$, and it includes the class of nondecreasing functions which are increasing beyond a certain point in its domain. For $w \in W$, we define:

$$
D(w)=\left\{\bar{c} \in \mathbb{R}_{+}: w(c)>w(\bar{c}) \text { for all } c>\bar{c}\right\} .
$$

These are points $(\bar{c})$ in the domain of $w$, such that increasing $c$ will lead to a higher felicity than at $\bar{c}$.

\subsection{Utilitarian Maximality}

The utilitarian social welfare relation that we use is based on the notion of weak-maximality used by Brock (1970) in the optimal growth literature. For $c, c^{\prime} \in C(\kappa)$, and $w \in W$, we write $c^{\prime} \succsim_{w} c$ iff:

$$
\liminf _{T \rightarrow \infty} \sum_{t=1}^{T}\left[w(c(t))-w\left(c^{\prime}(t)\right)\right] \leq 0 .
$$

A basic property of the social welfare relation $\succsim_{w}$ can be stated as follows.

Lemma 3 For $c, c^{\prime} \in C(\kappa)$, and $w \in W$, we have $c^{\prime} \succ_{w} c$ if and only if:

$$
\liminf _{T \rightarrow \infty} \sum_{t=1}^{T}\left[w\left(c^{\prime}(t)\right)-w(c(t))\right]>0 .
$$

Let $\bar{c} \in C(\kappa)$ and $w \in W$. Then $\bar{c}$ is called $w$-maximal if there is no $c \in C(\kappa)$ satisfying $c \succ_{w} \bar{c}$. Then, by Lemma 3, we see that given $\bar{c} \in C(\kappa)$ and $w \in W, \bar{c}$ is $w$-maximal if for every $c \in C(\kappa)$, we have:

$$
\liminf _{T \rightarrow \infty} \sum_{t=1}^{T}[w(c(t))-w(\bar{c}(t))] \leq 0 .
$$




\subsection{Utilitarian Maximality implies Suppes-Sen Maximality}

We are now in a position to state the main result of this section. If for some $w \in W$, we know that $\bar{c} \in C(\kappa)$ is w-maximal, then we can conclude that $\bar{c}$ is also S-maximal. This gives us a useful sufficient condition for obtaining S-maximal paths (which does not involve permutations). It is particularly oriented to applications because we have chosen the class $W$ to be very broad, and we only need to check w-maximality for a single element $w$ of this class.

Proposition 1 Let $\bar{c} \in C(\kappa)$, and suppose that for some $w \in W$, with $\bar{c}(N) \in D(w)$ for some $N \in \mathbb{N}$, it is $w$-maximal. Then, it is also S-maximal.

\section{Dynamic Properties of Suppes-Sen Maximal Paths}

In this section we investigate the dynamic properties of Suppes-Sen maximal paths in the standard aggregative growth model. That is, given a Suppes-Sen maximal path, we investigate the necessary conditions that it must satisfy, and we infer from these conditions the behavior of consumption and the capital stock over time. We are not only interested in the long-run or asymptotic behavior of these key economic magnitudes, but also in the transition dynamics.

In the first subsection below, we study the nature of S-maximal paths along which the capital stock is always below the golden-rule stock. In the second subsection, we examine the nature of S-maximal paths along which the capital stock is always above the golden-rule stock.

\subsection{Suppes-Sen Maximal Paths below the Golden-Rule}

We show below that if $\{k(t)\}$ is a Suppes-Sen maximal path, such that the output producible at each date on this path is less than or equal to the golden-rule stock, then its capital stock and consumption sequences must be monotone non-decreasing over time, with the capital stock sequence converging to a steady-state below the golden-rule stock. Thus, such Suppes-Sen maximal paths in the standard aggregative model behave like the Suppes-Sen maximal paths in the productive model examined in Asheim et al (2010).

We present this result by using the concept of "immediate productivity" introduced in Asheim, Buchholz and Tungodden (2001). ${ }^{13}$ To keep our exposition self-contained, we include a definition of it, which is a slight variant of the one used in their paper, but equivalent to it.

A feasible path $\{k(t), c(t+1)\}$ from $\kappa \geq 0$ satisfies immediate productivity if $c(s)>c(s+1)$ for some $s \geq 1$ implies the existence of a feasible path $\left\{k^{\prime}(t), c^{\prime}(t+1)\right\}$ from $\kappa$ with $c^{\prime}(s)=c(s+1), c^{\prime}(s+1)>c(s)$, and $c^{\prime}(t)=c(t)$ for all $t \neq s, s+1$.

We first show that any feasible path $\{k(t), c(t+1)\}$ from $\kappa \geq 0$, satisfying $f(k(t)) \leq z$ for all $t \geq 0$, satisfies immediate productivity.

Lemma 4 Suppose $\{k(t), c(t+1)\}$ is a feasible path from $\kappa \geq 0$, satisfying $f(k(t)) \leq z$ for $t \geq 0$. Then $\{k(t), c(t+1)\}$ satisfies immediate productivity.

Next, we show that if $\{k(t), c(t+1)\}$ is a feasible path from $\kappa \geq 0$ for which the capital stock never exceeds the golden-rule capital stock $z$, and for which consumption is monotone non-decreasing over time, then its capital stock sequence must also be non-decreasing over time, and converging to a capital stock not exceeding $z$. Note that for this result, it is not necessary to assume that the feasible path $\{k(t), c(t+1)\}$ satisfies $f(k(t)) \leq z$ for all $t \geq 0$.

\footnotetext{
${ }^{13}$ We thank an editor of this journal for suggesting to us this approach to the result, as well as for providing us with complete proofs of Lemma 4, Proposition 2 and Proposition 3 (which are reported in section 7). Our earlier approach used alternative proofs, which did not directly use the concept of immediate productivity.
} 
Proposition 2 Suppose $\{k(t), c(t+1)\}$ is a feasible path from $\kappa \geq 0$, satisfying $k(t) \leq z$ for $t \geq 0$, and $c(t+1) \leq c(t+2)$ for all $t \geq 0$. Then (i) $k(t) \leq k(t+1)$ for $t \geq 0$; and (ii) $\{k(t)\}$ converges to some $\bar{k}$ satisfying $\kappa \leq \bar{k} \leq z$.

Using Lemma 4 and Proposition 2, we can establish the following result on the behavior of the consumption and capital stock sequences along a Suppes-Sen maximal path.

Proposition 3 Suppose that $\{k(t)\}$ is a Suppes-Sen maximal path from $\kappa \geq 0$, satisfying $f(k(t)) \leq z$ for all $t \geq 0$. Then (i) $c(t+1) \leq c(t+2)$ for all $t \geq 0$; (ii) $k(t) \leq k(t+1)$ for $t \geq 0$; and (iii) $\{c(t)\}$ converges to $\bar{c}=f(\bar{k})-\bar{k}$, where $\bar{k}$ is given by part (ii) of Proposition 2 .

It is important to stress at this point that Proposition 3 applies to Suppes-Sen maximal paths $\{k(t)\}$, which satisfy $f(k(t)) \leq z$ for $t \geq 0$; it does not have anything to say about Suppes-Sen maximal paths $\{k(t)\}$, which satisfy $k(t) \leq z$ for $t \geq 0$, but fail to satisfy the condition $f(k(t)) \leq z$ for $t \geq 0$.

Proposition 3 uses the fact that a Suppes-Sen maximal path $\{k(t)\}$, which satisfies $f(k(t)) \leq z$ for $t \geq 0$, must satisfy immediate productivity (by Lemma 4), and therefore must also satisfy the property that its consumption sequence is monotonic non-decreasing (by the definition of Suppes-Sen maximality).

So, a natural question that arises is whether immediate productivity holds for every Suppes-Sen maximal path $\{k(t)\}$, which satisfies $k(t) \leq z$ for $t \geq 0$. For, if it does, then the result of Proposition 3 could be generalized to apply to all such paths.

Actually, immediate productivity does not hold for all Suppes-Sen maximal paths along which the capital stocks never exceed $z$, and the result of Proposition 3 does not generalize to all such paths.

This is best seen by considering a particular case of the example that has been fully developed by us in Section 5.2 below. Consider in that example that the parameters $a$ and $b$ are given by $a=2$ and $b=(1 / 2)$. Then, the golden-rule capital stock, $z$, is given by $z=1$. We show there that the capital stock sequence:

$$
\{k(t)\}=\{k(0), k(1), k(2), k(3), \ldots\}=\{1,(1 / 2), 1,(1 / 2), \ldots\}
$$

is a Suppes-Sen maximal path from the initial capital stock $k(0)=z=1$. Clearly, $\{k(t)\}$ is a path for which the capital stock never exceeds the golden-rule capital stock. The associated consumption sequence is given by:

$$
\{c(t+1)\}=\{c(1), c(2), c(3), c(4), \ldots\}=\{(3 / 2), 0,(3 / 2), 0, \ldots\} .
$$

This indicates that the monotonicity properties for capital stocks and consumption levels, obtained in Proposition 3, do not hold for all Suppes-Sen maximal paths which have capital stock sequences not exceeding the golden-rule.

To see that immediate productivity fails for the Suppes-Sen maximal path $\{k(t)\}$, we proceed as follows. Note that $c(1)>c(2)$. Immediate productivity would require that the consumption sequence:

$$
\left\{c^{\prime}(t+1)\right\}=\left\{c(2), c^{\prime}(2), c(3), c(4), \ldots\right\}=\left\{0, c^{\prime}(2),(3 / 2), 0, \ldots\right\}
$$

with $c^{\prime}(2)>(3 / 2)$ be generated by a feasible path $\left\{k^{\prime}(t)\right\}$ from $z=1$. But, this would imply that the sequence $\left\{k^{\prime}(t)\right\}$ must satisfy:

$$
k^{\prime}(0)=1, k^{\prime}(1)=2 \text { and } k^{\prime}(2)=f(2)-c^{\prime}(2)<f(2)-(3 / 2)=(5 / 2)-(3 / 2)=1
$$

Since $\left\{k^{\prime}(t)\right\}$ generates the sequence $\{c(3), c(4), \ldots\}$ starting from $k^{\prime}(2)<1$, and $\{k(t)\}$ generates the sequence $\{c(3), c(4), \ldots\}$ starting from $k(2)=1,\{k(t)\}$ must be inefficient. But, since $k(t) \leq z$ for all $t \geq 0$, it follows from the basic characterization result in Cass (1972, p.204-5) that $\{k(t)\}$ is efficient. This contradiction establishes that immediate productivity is violated for the Suppes-Sen maximal path $\{k(t)\}$. 


\subsection{Suppes-Sen Maximal Paths above the Golden-Rule}

Suppes-Sen maximal paths above the golden-rule stock are more difficult to analyze than paths below the golden-rule stock. This arises from two reasons: (i) the production function is not productive in this range, so the reasoning used for the productive range will not be applicable, but more important (ii) there is a lack of symmetry between paths above and below the golden rule stock, because paths above and bounded away from the golden rule stock are necessarily inefficient and hence cannot be Suppes-Sen maximal (while paths below the golden rule stock are necessarily efficient).

We start our analysis again with a Lemma, which gives a sufficient condition under which a path above the golden-rule stock will not be Suppes-Sen maximal.

Lemma 5 Suppose $\{k(t)\}$ is a feasible path from $\kappa \geq 0$, satisfying $k(t) \geq z$ for $t \geq 0$. If there is $s \geq 0$, such that:

$$
c(s+1)<c(s+2) \leq f(z)-z
$$

then $\{k(t)\}$ cannot be Suppes-Sen maximal.

We show using the Lemma that the capital stock sequence either (i) converges to the golden-rule capital stock, or (ii) generates "persistent fluctuations".

Proposition 4 Suppose $\{k(t)\}$ is a Suppes-Sen maximal path from $\kappa \geq 0$, satisfying $k(t) \geq z$ for $t \geq 0$. Then one of the following two possibilities will obtain:

(i) There is $T \in \mathbb{N}$ such that for all $t \geq T, k(t+1) \leq k(t)$, and $c(t+2) \leq c(t+1)$, and $(k(t), c(t)) \rightarrow$ $(z, f(z)-z)$ as $t \rightarrow \infty$;

(ii) There is an infinite sequence of periods $\left\{t_{1}, t_{2}, \ldots\right\}$ with $t_{1}<t_{2}<\cdots$, such that for every $s \in\left\{t_{1}, t_{2}, \ldots\right\}$, there are fluctuations in the capital stock of the following type:

$$
k(s)<k(s+1) \text { and } k(s+1)>k(s+2)
$$

and:

$$
c(s+2)>f(z)-z>c(s+1) .
$$

Remark 1 (i) If $w$ is any increasing and strictly concave function on $\mathbb{R}_{+}$, then for every $\kappa \geq z$, there is a unique feasible path $\{\bar{k}(t)\}$ from $\kappa$ satisfying:

$$
\liminf _{T \rightarrow \infty} \sum_{t=1}^{T}[w(c(t))-w(\bar{c}(t))] \leq 0
$$

for every feasible path $\{k(t)\}$ from $\kappa$. For such a path, we know that for all $t \geq 0, k(t+1) \leq k(t)$, and $c(t+2) \leq c(t+1)$, and $(k(t), c(t)) \rightarrow(z, f(z)-z)$ as $t \rightarrow \infty$. Since we also know (from Proposition 1) that such a path is Suppes-Sen maximal, possibility (i) in Proposition 4 can actually occur for Suppes-Sen maximal paths.

(ii) It would be useful to have an example in which possibility (ii) in Proposition 4 actually occurs for a Suppes-Sen maximal path. We now present an example to illustrate this possibility.

Example 1: The production function, $f$, is piecewise linear and specified by two parameters $\{a, b\}$, with $a>1>b$ and:

$$
a b>1+b .
$$

For a numerical example, consider $a=4$ and $b=(1 / 2)$. The function, $f$, is defined as follows:

$$
f(x)= \begin{cases}a x & \text { for } x \in[0,1] \\ a+b(x-1) & \text { for } x>1\end{cases}
$$


The golden-rule stock, $z$, is equal to 1 , and the maximum sustainable stock, $K$, is $(a-b) /(1-b)$. The golden-rule consumption is $(a-1)$. Denote the stock level $f(a) \equiv[a+b(a-1)]$ by $q$ and the consumption level $f(f(z))-z=f(a)-1=(a-1)(b+1)$ by $\theta$. Note that $f(f(x))-x$ is maximized at $x=z$ among all $x \geq 0$. Thus,

$$
f(f(x))-x \leq \theta \text { for all } x \geq 0 .
$$

To verify this, suppose on the contrary there is some $x \geq 0$ such that $f(f(x))-x>\theta$. Then, since $\theta=$ $f(a)-1=a+b(a-1)-1>a$, by Condition (C), we must have $f(x)>1$. Then, if $x \leq 1$,

$$
\begin{aligned}
f(f(x))-x & =a+b[f(x)-1]-x=(a-b)+b f(x)-x=(a-b)+b a x-x \\
& =(a-b)+(a b-1) x \leq(a-b)+(a b-1)=\theta
\end{aligned}
$$

a contradiction. And, if $x>1$, then:

$$
\begin{aligned}
f(f(x))-x & =(a-b)+b f(x)-x=(a-b)+b[a+b(x-1)]-x \\
& =(a-b)+a b-b^{2}-\left(1-b^{2}\right) x<(a-b)+a b-b^{2}-\left(1-b^{2}\right)=\theta
\end{aligned}
$$

a contradiction.

Define $w$ by:

$$
w(c)= \begin{cases}0 & \text { for } c \in[0, \theta) \\ c & \text { for } c \geq \theta .\end{cases}
$$

Clearly, $w$ is not concave, not increasing (on $[0, \theta)$ ) and not continuous (at $\theta$ ). However, note that $w \in W$, and $D(w)=[\theta, \infty)$. [We defined the class $W$ to be broad enough to accommodate such felicity functions]. A useful property of $w$ is that $w(c) \leq c$ for all $c \geq 0$.

Define the transition possibility set:

$$
\Omega=\left\{\left(x, x^{\prime}\right) \in \mathbb{R}_{+}^{2}: x^{\prime} \leq f(x)\right\}
$$

and a reduced form utility function $u: \Omega \rightarrow \mathbb{R}_{+}$by:

$$
u\left(x, x^{\prime}\right)=w\left(f(x)-x^{\prime}\right) \text { for all }\left(x, x^{\prime}\right) \in \Omega .
$$

The principal technical result that we will need is the following price support lemma for a period two cycle.

Lemma 6 If $\left(x, x^{\prime}\right) \in \Omega$, and $\left(x^{\prime}, x^{\prime \prime}\right) \in \Omega$, then

$$
u\left(x, x^{\prime}\right)+u\left(x^{\prime}, x^{\prime \prime}\right)+x^{\prime \prime}-x \leq u(z, a)+u(a, z) \equiv \theta .
$$

It is now straightforward to verify that the sequence $\{z, f(z), z, f(z), \ldots\}$ is a feasible path from $z$, with associated consumption sequence $\{0, \theta, 0, \theta, \ldots\}$. Using Lemma 6 , it can be shown ${ }^{14}$ that this path is SuppesSen maximal from $z$.

\section{Suppes-Sen Maximality and Consumption Cycles}

Example 1 of Section 4.2 demonstrates that Suppes-Sen maximality can lead to the choice of a growth path which is cyclical in consumption. We strengthen this finding in a particularly compelling way by obtaining explicitly a consumption function, which (a) generates only Suppes-Sen maximal paths, and (b) all such

\footnotetext{
${ }^{14}$ The method involved is elaborated in the proof of Theorem 1, part (A), case (ii), and is therefore not repeated here. See Section 5.2.5 and Section 7 below.
} 
Suppes-Sen maximal paths exhibit a period-two consumption cycle after at most a finite number of time periods. Such a consumption cycle involves a capital stock sequence which alternates between the goldenrule stock and a stock below the golden-rule stock.

The consumption function is derived by solving for w-maximal paths for a particular choice of $w \in W$, thereby showing the usefulness of the results of Section 3. The nature of the consumption function is such that it leads to consumption cycles, and this aspect can be best understood by recognizing that this is to be expected if we solve for w-maximal paths for a choice of $w \in W$, which is suitably non-concave.

It is useful to point out that studying w-maximality with a non-concave $w \in W$ is not the objective of this study. It is only a device which enables us to obtain an S-maximal consumption function (by relying on Proposition 1, which establishes the connection between the two concepts). However, it is also useful to recognize that the study of S-maximality leads very naturally to the study of w-maximality with $w \in W$ which are not necessarily concave.

Studying w-maximality with a $w \in W$ which is not concave is, of course, far from straightforward. Almost all the literature on (undiscounted) optimal growth deals with w-maximality (or one of its variants) for $w \in W$, which is also concave (mostly, strictly concave). However, as this section shows, the available techniques can be suitably modified to study w-maximality with the specific non-concave $w \in W$ that we use. We hope that these methods can be used in other contexts which involve dynamic optimization over an infinite horizon with a not necessarily concave return function.

\subsection{S-Maximal Consumption Function}

A consumption function is a rule $h:[0, K] \rightarrow[0, K]$, assigning a consumption choice $h(y) \in[0, K]$ for every output level $y \in[0, K]$, such that $h(y) \leq y$. Note that a consumption function is time-independent. Also, the consumption choice $h(y)$ depends only on the output level $y$, and not on aspects like how and when the output level $y$ is attained. It says that, whenever the output level is $y$, the consumption choice is $h(y)$.

A consumption function $h$ generates a feasible path $\{k(t)\}$ starting from any $\kappa \in[0, K]$ as follows:

$$
k(0)=\kappa, k(t+1)=f(k(t))-h(f(k(t))) \text { for all } t \in \mathbb{N}_{0} .
$$

Its associated consumption sequence $\{c(t+1)\}$ is given by:

$$
c(t+1)=h(f(k(t))) \text { for all } t \in \mathbb{N}_{0} .
$$

An $S$-maximal consumption function is a consumption function, $h$, such that for every $\kappa \in[0, K]$, the consumption sequence associated with the feasible path generated by $h$ is S-maximal in $C(\kappa)$.

\subsection{An Example}

We now analyze in detail a specific example of the framework described in Section 2, in the context of which we solve for an S-maximal consumption function.

\subsubsection{Specification of Consumption Possibilities}

The production function, $f$, is piecewise linear and specified by two parameters $\{a, b\}$, with $a>1$ and $a b=1$. For a numerical example, consider $a=2$ and $b=(1 / 2)$. The function, $f$, is defined as follows:

$$
f(x)= \begin{cases}a x & \text { for } x \in[0,1] \\ a+b(x-1) & \text { for } x>1\end{cases}
$$

The golden-rule stock, $z$, is equal to 1 , and the maximum sustainable stock, $K$, is $(a-b) /(1-b)$. The golden-rule consumption is $(a-1)$. 
Define the transition possibility set, $\Omega$, by:

$$
\Omega=\left\{\left(x, x^{\prime}\right) \in \mathbb{R}_{+}^{2}: x^{\prime} \leq f(x)\right\}
$$

and the two-period transition possibility set, $\Lambda$, by:

$$
\Lambda=\left\{\left(x, x^{\prime \prime}\right) \in \mathbb{R}_{+}^{2}: \text { there is } x^{\prime} \in \mathbb{R}_{+} \text {, such that }\left(x, x^{\prime}\right) \in \Omega \text { and }\left(x^{\prime}, x^{\prime \prime}\right) \in \Omega\right\} .
$$

For $\left(x, x^{\prime \prime}\right) \in \Lambda$, we also define $\Gamma\left(x, x^{\prime \prime}\right)=\left\{x^{\prime} \in \mathbb{R}_{+}:\left(x, x^{\prime}\right) \in \Omega\right.$ and $\left.\left(x^{\prime}, x^{\prime \prime}\right) \in \Omega\right\}$.

\subsubsection{Specification of Felicity Function}

Denote $(a-b)$ by $\theta$, and define $w$ by:

$$
w(c)= \begin{cases}0 & \text { for } c \in[0, \theta) \\ c & \text { for } c \geq \theta .\end{cases}
$$

Clearly, $w$ is not concave, not increasing (on $[0, \theta)$ ) and not continuous (at $\theta$ ). However, note that $w \in W$, and $D(w)=[\theta, \infty)$. A useful property of $w$ is that $w(c) \leq c$ for all $c \geq 0$.

Given the felicity function $w$, we define a reduced-form utility function $u: \Omega \rightarrow \mathbb{R}$ by:

$$
u\left(x, x^{\prime}\right)=w\left(f(x)-x^{\prime}\right) \text { for all }\left(x, x^{\prime}\right) \in \Omega .
$$

\subsubsection{Price Support of a Period Two Cycle}

It should be clear that, with the chosen felicity function $w$, the golden-rule loses its traditional importance in optimal growth models. Staying at the golden-rule forever cannot be w-maximal. Since the golden rule consumption is $(a-1)<(a-b) \equiv \theta$, and so $w(a-1)=0$, the felicity sequence along the golden-rule path is $\{0,0,0, \ldots\}$, while the feasible path $\{1, b, 1, b, \ldots\}$ from the golden-rule stock yields the felicity sequence $\{\theta, 0, \theta, 0, \ldots\}$.

The central importance of the golden-rule and its price support is usurped by a specific period two cycle and its price support. This makes the traditional methods still applicable by viewing the period two cycle as stationary in terms of the two-period transition possibility set, $\Lambda$.

The specific cycle of interest is specified by the stock sequence $\{b, 1, b, 1, \ldots\}$, and associated consumption sequence $\{0, \theta, 0, \theta, \ldots\}$. The stationary price support for it is $p=1$. The following Lemma (similar to Lemma 6), summarizing this result, is crucial to much of the subsequent analysis.

Lemma 7 Let $\left(x, x^{\prime}\right) \in \Omega$, and $\left(x^{\prime}, x^{\prime \prime}\right) \in \Omega$. Then,

$$
u\left(x, x^{\prime}\right)+u\left(x^{\prime}, x^{\prime \prime}\right)+x^{\prime \prime}-x \leq \theta=u(b, 1)+u(1, b) .
$$

\subsubsection{Value Losses}

In the standard theory of optimal intertemporal allocation, when future utilities are not discounted, and the felicity function is increasing and strictly concave, the nature of (catching-up) optimal paths can be examined by focusing on value losses suffered by a path for not operating at the golden-rule. In our context, given the price support of the period two cycle in Lemma 7, it is natural to focus instead on value losses suffered by a path for not operating at the period-two cycle, and this is precisely what we do.

For $\left(x, x^{\prime \prime}\right) \in \Lambda$, we can define:

$$
R\left(x, x^{\prime \prime}\right)=\sup _{x^{\prime} \in \Gamma\left(x, x^{\prime \prime}\right)}\left\{u\left(x, x^{\prime}\right)+u\left(x^{\prime}, x^{\prime \prime}\right)+x^{\prime \prime}-x\right\} .
$$


By Lemma 7, we know that $R\left(x, x^{\prime \prime}\right) \leq \theta$ for all $\left(x, x^{\prime \prime}\right) \in \Lambda$. We define the value-loss from operating at $\left(x, x^{\prime \prime}\right) \in \Lambda$ by:

$$
L\left(x, x^{\prime \prime}\right)=\theta-R\left(x, x^{\prime \prime}\right) .
$$

Clearly, $L\left(x, x^{\prime \prime}\right) \geq 0$ for all $\left(x, x^{\prime \prime}\right) \in \Lambda$. We now establish a version of the value-loss lemma of Radner (1961), Atsumi (1965) and McKenzie (1968) for our framework. This ensures that being uniformly away from the period-two cycle produces a uniform value loss.

Lemma 8 Given any $\alpha \in(0, b]$, there is $\beta>0$, such that whenever $\left(x, x^{\prime \prime}\right) \in \Lambda$, and $(b-x) \geq \alpha$, we have:

$$
L\left(x, x^{\prime \prime}\right) \geq \beta
$$

\subsubsection{S-maximal Consumption Function}

The price-support property of the period two cycle in Lemma 7, and the value-loss property of Lemma 8, enable us to use suitable modifications of standard arguments in the theory of undiscounted (catching-up) optimal growth and show that the consumption function, $h:[0, K] \rightarrow[0, K]$, defined by:

$$
h(y)= \begin{cases}0 & \text { for } y \in[0, a) \\ y-b & \text { for } y \in[a, K]\end{cases}
$$

is a $w$-maximal consumption function for the $w$ specified in $\left(\mathrm{W}^{\prime}\right)$, and therefore an $\mathrm{S}$-maximal consumption function (by Proposition 1).

The consumption function $h$ leads to the following dynamical system, governing the behavior over time of the capital stock sequence $\{k(t)\}$ generated by it, starting from any initial stock $\kappa \in[0, K]$ :

$$
k(0)=\kappa ; k(t+1)= \begin{cases}a k(t) & \text { for } k(t) \in[0,1) \\ b & \text { for } k(t) \in[1, K]\end{cases}
$$

Thus, starting from any initial stock $\kappa \in(0, K]$, the capital stock sequence $\{k(t)\}$ generated by the S-maximal consumption function $h$ coincides with the period two cycle $\{b, 1, b, 1, \ldots\}$ after at most a finite number of periods. This means that after at most a finite number of periods, the feasible path generated by the S-maximal consumption function $h$ exhibits the consumption cycle $\{0, \theta, 0, \theta, \ldots\}$. We summarize these findings in the following theorem.

Theorem 1 (A) The consumption function h, specified in $(C F)$, is (a) a w-maximal consumption function for the $w$ specified in $\left(W^{\prime}\right)$, and $(b)$ an $S$-maximal consumption function. That is, every path generated by $h$ is a Suppes-Sen maximal path.

(B) Every path from $\kappa \in(0, K]$, generated by the consumption function $h$, exhibits period-two consumption cycles after at most a finite number of time periods.

\subsubsection{Pigou-Dalton Transfer Principle}

Following Asheim et al (2010), we will say that a consumption function $h$ satisfies the Pigou-Dalton transfer principle if for all $\kappa \in[0, K]$, and for all non-negative sequences $\{c(t+1)\}_{0}^{\infty},\left\{c^{\prime}(t+1)\right\}_{0}^{\infty}$, (i) if there exist $\varepsilon>0$ and $S, T \in \mathbb{N}_{0}$ such that $c(S+1)=c^{\prime}(S+1)-\varepsilon \geq c^{\prime}(T+1)+\varepsilon=c(T+1)$, and $c(t+1)=c^{\prime}(t+1)$ for all $t \in \mathbb{N}_{0} /\{S, T\}$, and further (ii) $\left\{c^{\prime}(t+1)\right\}_{0}^{\infty}$ is the consumption sequence generated by the consumption function $h$ starting with the initial capital stock $\kappa$, then $\{c(t+1)\}_{0}^{\infty}$ cannot be a consumption sequence associated with a feasible path starting from $\kappa$.

We now show that for the production function $f$ given by $\left(\mathrm{PF}^{\prime}\right)$, the consumption function $h$ given by (CF) does not satisfy the Pigou-Dalton transfer principle. 
Consider the feasible path $\left\{k^{\prime}(t)\right\}$ from $\kappa=1$, defined by the sequence of capital stocks $\{1, b, 1, b, \ldots\}$, with associated consumption sequence $\left\{c^{\prime}(t+1)\right\}$ given by $\{\theta, 0, \theta, 0, \ldots\}$. It is easy to check that $\left\{c^{\prime}(t+1)\right\}$ is the consumption sequence generated by the consumption function $h$ starting with the initial capital stock $\kappa=1$. Define the sequence $\{k(t)\}$ from $\kappa=1$ as follows:

$$
\left.\begin{array}{l}
k(0)=k=1 ; k(1)=b+\varepsilon ; k(2)=1+(a-1) \varepsilon \\
k(t+1)=f(k(t))-c^{\prime}(t+1) \quad \text { for all } t \geq 2
\end{array}\right\}
$$

where $0<\varepsilon=(1-b) / 2<(a-b) / 2$. Clearly $k(1)>k^{\prime}(1)$ and $k(2)>k^{\prime}(2)$. It is now easy to verify recursively from the last line of (13) that $k(t)>k^{\prime}(t)$ for all $t>2$. Further, since $f(k(0))=f(1)=a$, and $k(1)=b+\varepsilon$, we have $f(k(0))-k(1)=(a-b)-\varepsilon=c^{\prime}(1)-\varepsilon>0$. And since $k(1)<b+[(a-b) / 2]<a$, we have $f(k(1))-k(2)=(1+a \varepsilon)-[1+(a-1) \varepsilon]=\varepsilon=c^{\prime}(2)+\varepsilon>0$. Thus, using the last line of (13), it follows that $\{k(t)\}$ is a feasible path from $\kappa=1$, with associated consumption sequence $\{c(t+1)\}$ given by $c(1)=c^{\prime}(1)-\varepsilon, c(2)=c^{\prime}(2)+\varepsilon$, and $c(t+1)=c^{\prime}(t+1)$ for all $t \geq 2$. By choice of $\varepsilon$, we have:

$$
c(1)=c^{\prime}(1)-\varepsilon=(a-b)-\varepsilon>\varepsilon=c^{\prime}(2)+\varepsilon=c(2) .
$$

Thus, $h$ violates the Pigou-Dalton transfer principle, by using $S=0$ and $T=1$.

\section{Concluding Remarks}

In this paper, we have undertaken a systematic study of growth paths in the canonical aggregative model of economic growth, which are maximal according to the Suppes-Sen grading principle. Since the grading principle embodies two leading principles, equity and efficiency, in the theory of social choice, our study should be helpful in guiding intertemporal decision making with respect to consumption and savings.

We are able to describe precisely the dynamic properties of Suppes-Sen maximal paths for which capital stocks always lie above or always lie below the golden-rule stock. These include establishing monotonicity and convergence properties, as well as demonstrating the possibility of consumption cycles.

Our characterization of Suppes-Sen maximal paths, however, remains incomplete. The leading open question arising from this study is to find such a characterization, and compare it with the characterization of paths which are maximal with respect to the efficiency quasi-ordering (a less complete quasi-ordering than the grading principle), and those which are maximal with respect to the catching up optimality quasiordering (a more complete quasi-ordering than the grading principle).

Our paper also makes a useful contribution in suggesting a method to analyze Suppes-Sen maximal paths. This is accomplished by establishing a link between paths which are Suppes-Sen maximal and those which are weakly maximal in terms of the utilitarian criterion, for a class of non-decreasing (but not necessarily concave) felicity functions. We hope that this method can be exploited to obtain a complete characterization of Suppes-Sen maximal paths. It might also be useful in other contexts which involve dynamic optimization over an infinite horizon with a not necessarily concave return function.

\section{Appendix: Proofs}

Proof of Lemma 1. Let $T=1$. Since $f(0)=0$, and $c(1)>0$, we have $k(0)>0$. Since $f$ is continuous, we can pick $\varepsilon(1)>0$ (sufficiently close to 0 ) to ensure that $\varepsilon(1)<c(1)-\gamma$. For $t \geq 1$, define iteratively:

$$
\varepsilon(t+1)=(1 / 2)[f(k(t)+\varepsilon(t))-f(k(t))] .
$$

Then, $\{\varepsilon(t)\}$ is well-defined, and $\varepsilon(t)>0$ for $t \geq 1$, since $f$ is increasing. Now define $k^{\prime}(0)=k(0)$, and $k^{\prime}(t)=k(t)+\varepsilon(t)$ for $t \geq 1$. Then,

$$
f(k(0))-k^{\prime}(1)=f(k(0))-k(1)-\varepsilon(1)=c(1)-\varepsilon(1)>\gamma
$$


and for $t \geq 1$,

$$
\begin{aligned}
f\left(k^{\prime}(t)\right)-k^{\prime}(t+1) & =f(k(t)+\varepsilon(t))-k(t+1)-\varepsilon(t+1) \\
& =f(k(t)+\varepsilon(t))-f(k(t))+f(k(t))-k(t+1)-\varepsilon(t+1) \\
& =c(t+1)+\varepsilon(t+1) .
\end{aligned}
$$

Clearly, (14) and (15) establish that $\left\{k^{\prime}(t)\right\}$ is a feasible path from $\kappa$, and that its associated consumption sequence $\left\{c^{\prime}(t+1)\right\}$ satisfies $c^{\prime}(1)>\gamma$, and $c^{\prime}(t+1)>c(t+1)$ for all $t \neq 0$.

The case $T>1$ can be handled similarly in the forward direction, and with suitable decreases in the stock sequence in the backward direction.

Proof of Corollary 1. Since $c$ is not S-maximal, there is $c^{\prime} \in C(\kappa)$, and $\pi \in \mathcal{F}$, such that:

$$
c^{\prime} \circ \pi>c .
$$

Using (16), there is a time period, $\tau$, for which $c^{\prime}(\pi(\tau))>c(\tau)$. Denote $\pi(\tau)$ by $T$, and $c(\tau)$ by $\gamma$. Then, using Lemma 1, there is a feasible path $\{\bar{k}(t)\}$ from $\kappa$, with associated consumption sequence $\{\bar{c}(t)\}$, such that $\bar{c}(t)>c^{\prime}(t)$ for all $t \neq T$, and $\bar{c}(T)>\gamma \equiv c(\tau)$. Then, for every $s \in \mathbb{N}$, with $s \neq \tau$, we have $\bar{c}(\pi(s))>$ $c^{\prime}(\pi(s)) \geq c(s)$ by (16). And, for $s=\tau$, we have $\bar{c}(\pi(s))=\bar{c}(T)>\gamma=c(\tau)=c(s)$. This establishes (3).

Proof of Lemma 3. If $c^{\prime} \succ_{w} c$, then $c^{\prime} \succsim_{w} c$ holds and $c \succsim_{w} c^{\prime}$ does not hold. The former restriction implies that (4) holds. The latter restriction implies that the following inequality does not hold:

$$
\liminf _{T \rightarrow \infty} \sum_{t=1}^{T}\left[w\left(c^{\prime}(t)\right)-w(c(t))\right] \leq 0 .
$$

Thus, (5) must hold.

To go in the opposite direction, let $c, c^{\prime} \in C(\kappa), w \in W$, and suppose (5) holds. This means:

$$
\liminf _{T \rightarrow \infty} \sum_{t=1}^{T}\left[w(c(t))-w\left(c^{\prime}(t)\right)\right] \leq \limsup _{T \rightarrow \infty} \sum_{t=1}^{T}\left[w(c(t))-w\left(c^{\prime}(t)\right)\right]=-\liminf _{T \rightarrow \infty} \sum_{t=1}^{T}\left[w\left(c^{\prime}(t)\right)-w(c(t))\right]<0 .
$$

Clearly (17) means that $c^{\prime} \succsim_{w} c$, and (5) means that $c \succsim_{w} c^{\prime}$ does not hold. Thus, we have $c^{\prime} \succ_{w} c$.

Proof of Proposition 1. Suppose, on the contrary, there is some $c \in C(\kappa)$, such that $c \succ_{S} \bar{c}$. Then, there is $\pi \in \mathcal{F}$, such that $c \circ \pi>\bar{c}$. By Corollary 1 , there is $c^{\prime} \in C(\kappa)$ such that $c^{\prime} \circ \pi>>\bar{c}$. Since $\pi \in \mathcal{F}$, we can pick $N^{\prime} \in \mathbb{N}$, such that $N^{\prime} \geq N$, and $\pi(t)=t$ for all $t \geq N^{\prime}$. Then, we must have:

$$
\left(c^{\prime}(\pi(1)), \ldots, c^{\prime}\left(\pi\left(N^{\prime}\right)\right)\right)>>\left(\bar{c}(1), \ldots, \bar{c}\left(N^{\prime}\right)\right)
$$

and:

$$
c^{\prime}(t)=c^{\prime}(\pi(t))>\bar{c}(t) \text { for all } t>N^{\prime} .
$$

Since $\pi(t)=t$ for all $t \geq N^{\prime}, \pi$ must map $\left\{1, \ldots, N^{\prime}\right\}$ onto $\left\{1, \ldots, N^{\prime}\right\}$. Since $w \in W, N \leq N^{\prime}$, and $\bar{c}(N) \in D(w)$, it follows from (18) that:

$$
\sum_{t=1}^{N^{\prime}} w\left(c^{\prime}(t)\right)=\sum_{t=1}^{N^{\prime}} w\left(c^{\prime}(\pi(t))>\sum_{t=1}^{N^{\prime}} w(\bar{c}(t)) .\right.
$$

And, since $W \subset W_{N D}$, it follows from (19) that for all $T>N^{\prime}$,

$$
\sum_{t=N^{\prime}+1}^{T} w\left(c^{\prime}(t)\right) \geq \sum_{t=N^{\prime}+1}^{T} w(\bar{c}(t))
$$


Denoting $\left[\sum_{t=1}^{N^{\prime}} w\left(c^{\prime}(t)\right)-\sum_{t=1}^{N^{\prime}} w(\bar{c}(t))\right]$ by $\alpha$, we have $\alpha>0$. Using (20), we have for all $T>N^{\prime}$ :

$$
\sum_{t=1}^{T}\left[w\left(c^{\prime}(t)\right)-w(\bar{c}(t))\right] \geq\left[\sum_{t=1}^{N^{\prime}} w\left(c^{\prime}(t)\right)-\sum_{t=1}^{N^{\prime}} w(\bar{c}(t))\right]=\alpha
$$

which contradicts the w-maximality of $\bar{c}$.

Proof of Lemma 4. Suppose $\{k(t), c(t+1)\}$ is a feasible path from $\kappa \geq 0$ satisfying $f(k(t)) \leq z$ for $t \geq 0$. If $c(s)>c(s+1)$ for some $s \geq 1$, then the path $\left\{k^{\prime}(t), c^{\prime}(t+1)\right\}$ with $k^{\prime}(s)=k(s)+c(s)-c(s+1)>$ $k(s), k^{\prime}(t)=k(t)$ for $t \neq s, c^{\prime}(s)=c(s+1), c^{\prime}(s+1)=f\left(k^{\prime}(s)\right)-k(s+1)$ and $c^{\prime}(t)=c(t)$ for $t \neq s, s+1$, is feasible from $\kappa$. Observe that $k^{\prime}(s) \leq z$ as $c(s+1) \geq 0$ and $k(s)+c(s)=f(k(s-1)) \leq z$.

It remains to be shown that $c^{\prime}(s+1)>c(s)$.

$$
\begin{aligned}
c^{\prime}(s+1)-c(s) & =f\left(k^{\prime}(s)\right)-k(s+1)-c(s)=f\left(k^{\prime}(s)\right)-k^{\prime}(s)+k^{\prime}(s)-k(s+1)-c(s) \\
& =f\left(k^{\prime}(s)\right)-k^{\prime}(s)+k(s)+c(s)-c(s+1)-k(s+1)-c(s) \\
& =f\left(k^{\prime}(s)\right)-k^{\prime}(s)+k(s)-c(s+1)-k(s+1) \\
& =\left[f\left(k^{\prime}(s)\right)-k^{\prime}(s)\right]-[f(k(s))-k(s)]>0
\end{aligned}
$$

where the strict inequality in the last line of (21) follows from (2). This establishes the desired result.

Proof of Proposition 2. Suppose $\{k(t), c(t+1)\}$ is a feasible path from $\kappa \geq 0$ satisfying $k(t) \leq z$ and $c(t+1) \leq c(t+2)$ for $t \geq 0$. If $k(s-1)>k(s)$ for some $s \geq 1$, then:

$$
\begin{aligned}
c(s+1)+k(s) & \geq c(s)+k(s)=f((k(s-1)) \\
& >f(k(s))=c(s+1)+k(s+1)
\end{aligned}
$$

since $f$ is increasing. It follows from (22) that $k(s)>k(s+1)$, and by induction $k(t)>k(t+1)$ for all $t \geq s$. Since $k(t) \leq z$ for all $t \geq 0,\{k(t)\}$ must converge to some $\hat{k}$, and so $\{c(t+1)\}$ must converge to $\hat{c} \equiv f(\hat{k})-\hat{k}$. Note that we must have $\hat{k}<k(s-1)$, and $\hat{c} \geq c(s)$, since $\{k(t)\}$ is monotonic decreasing for $t \geq s-1$, and $\{c(t+1)\}$ is monotonic non-decreasing for all $t \geq 0$. However, we then obtain:

$$
\begin{aligned}
\hat{c} & =f(\hat{k})-\hat{k}<f(k(s-1))-k(s-1) \\
& <f(k(s-1))-k(s)=c(s)
\end{aligned}
$$

a contradiction. The strict inequality in the first line of (23) follows from (2). This establishes part (i). Part (ii) follows directly from part (i), since $k(t) \leq z$ for $t \geq 0$.

Proof of Proposition 3. Part (i) follows from Lemma 4, since if $c(s)>c(s+1)$ for some $s \geq 1$, then the feasible path $\left\{k^{\prime}(t), c^{\prime}(t+1)\right\}$ from $\kappa$, defined in the proof of Lemma 4, dominates a permutation of $\{k(t), c(t+1)\}$. Since $f(k(t)) \leq z$ for $t \geq 0$, we have $k(t) \leq z$ for $t \geq 0$, and Proposition 2 implies that (a) $k(t) \leq k(t+1)$ for $t \geq 0$; and (b) $\{k(t)\}$ converges to $\bar{k}$ as given by Proposition 2. This establishes parts (ii) and (iii).

Proof of Lemma 5. Define the sequence $\left\{k^{\prime}(t)\right\}$ by $k^{\prime}(t)=k(t)$ for all $t \neq s+1$, and:

$$
k^{\prime}(s+1)=f(k(s))-c(s+2) \text {. }
$$

Then, we have:

$$
k^{\prime}(s+1)=f(k(s))-c(s+2) \geq f(z)-(f(z)-z)=z .
$$

Further,

$$
f\left(k^{\prime}(s)\right)-k^{\prime}(s+1)=f(k(s))-[f(k(s))-c(s+2)]=c(s+2) \geq 0
$$

and:

$$
f\left(k^{\prime}(t)\right)-k^{\prime}(t+1)=f(k(t))-k(t+1)=c(t+1)
$$


for all $t \neq s, s+1$. We now show that:

$$
f\left(k^{\prime}(s+1)\right)-k^{\prime}(s+2)>c(s+1) .
$$

To this end, first note that:

$$
k^{\prime}(s+1)=f(k(s))-c(s+2)<f(k(s))-c(s+1)=k(s+1) .
$$

Using (24) and (28), we can write:

$$
\begin{aligned}
f\left(k^{\prime}(s+1)\right)-k^{\prime}(s+2) & =f\left(k^{\prime}(s+1)\right)-f(k(s+1))+f(k(s+1))-k(s+2) \\
& \left.>\left[k^{\prime}(s+1)\right)-k(s+1)\right]+f(k(s+1))-k(s+2) \\
& =[f(k(s))-c(s+2)-k(s+1)]+f(k(s+1))-k(s+2)=c(s+1)
\end{aligned}
$$

where the strict inequality in the second line follows from (2). This establishes (27). Using (25), (26) and (27), $\left\{k^{\prime}(t)\right\}$ is a feasible path from $\kappa$, with associated consumption sequence $\left\{c^{\prime}(t+1)\right\}$ given by $c^{\prime}(t+1)=f\left(k^{\prime}(t)\right)-k^{\prime}(t+1)$ for all $t \geq 0$. Further, (25) and (27) imply that $\{k(t)\}$ cannot be Suppes-Sen maximal.

Proof of Proposition 4. Suppose $\{k(t)\}$ is a Suppes-Sen maximal path from $\kappa \geq 0$, satisfying $k(t) \geq z$ for $t \geq 0$. Then clearly one of the following possibilities must be valid: (i) there is $T \in \mathbb{N}$, such that $c(t+2) \leq c(t+1)$ for all $t \geq T$; (ii) there is an infinite sequence of time periods $\left\{t_{1}, t_{2}, \ldots\right\}$ with $t_{1}<t_{2}<\cdots$, such that for every $s \in\left\{t_{1}, t_{2}, \ldots\right\}$, we have $c(s+2)>c(s+1)$.

We consider possibility (i) first. In this case, we have:

$$
f(k(t+1))-k(t+2) \leq f(k(t))-k(t+1) \text { for all } t \geq T .
$$

If $k(\tau+1)>k(\tau)$ for some $\tau \geq T$, then (29) implies:

$$
f(k(\tau))-k(\tau+2)<f(k(\tau+1))-k(\tau+2) \leq f(k(\tau))-k(\tau+1)
$$

so that $k(\tau+2)>k(\tau+1)$. Then the above step can be repeated to obtain $\{k(t)\}$ increasing for all $t \geq \tau$. Thus, using $k(\tau+1)>k(\tau) \geq z$, we get:

$$
\liminf _{t \rightarrow \infty} k(t) \geq k(\tau+1)>z
$$

But (30) implies that $\{k(t)\}$ is inefficient, which contradicts Suppes-Sen maximality of $\{k(t)\}$. Thus, (29) implies that:

$$
k(t+1) \leq k(t) \text { for all } t \geq T .
$$

It follows from (29) and (31) that $\{k(t)\},\{c(t)\}$ are convergent sequences. Since $k(t) \geq z$ for all $t \geq 1$, $\lim _{t \rightarrow \infty} k(t) \geq z$. Since $\{k(t)\}$ is efficient, $\lim _{t \rightarrow \infty} k(t)=z$. It follows that $\lim _{t \rightarrow \infty} c(t)=f(z)-z$. This establishes part (i) of the Proposition.

Now, consider possibility (ii). In this case, by Lemma 5, we must have $c(s+2)>f(z)-z$ for all $s \in\left\{t_{1}, t_{2}, \ldots\right\}$. If $k(s+2) \geq k(s+1)$ for some $s \in\left\{t_{1}, t_{2}, \ldots\right\}$, then we get:

$$
f(k(s+1))-k(s+1) \geq f(k(s+1))-k(s+2)>f(z)-z
$$

which contradicts the definition of $z$ as the golden-rule stock. Thus:

$$
k(s+2)<k(s+1) \text { for all } s \in\left\{t_{1}, t_{2}, \ldots .\right\} .
$$


We now proceed to verify that:

$$
k(s)<k(s+1) \text { for all } s \in\left\{t_{1}, t_{2}, \ldots\right\} .
$$

For if $k(s) \geq k(s+1)$ for some $s \in\left\{t_{1}, t_{2}, \ldots.\right\}$, then we can define the sequence $\left\{k^{\prime}(t)\right\}$ by $k^{\prime}(t)=k(t)$ for all $t \neq s+1$, and:

$$
k^{\prime}(s+1)=f(k(s))-c(s+2) .
$$

It follows that:

$$
k^{\prime}(s+1)=f(k(s))-c(s+2) \geq f(k(s+1))-c(s+2)=k(s+2) \geq z .
$$

Further,

$$
f\left(k^{\prime}(s)\right)-k^{\prime}(s+1)=f(k(s))-[f(k(s))-c(s+2)]=c(s+2) \geq 0
$$

and:

$$
f\left(k^{\prime}(t)\right)-k^{\prime}(t+1)=f(k(t))-k(t+1)=c(t+1)
$$

for all $t \neq s, s+1$.

We now show that:

$$
f\left(k^{\prime}(s+1)\right)-k^{\prime}(s+2)>c(s+1) .
$$

To this end, first note that:

$$
k^{\prime}(s+1)=f(k(s))-c(s+2)<f(k(s))-c(s+1)=k(s+1) .
$$

Using (35) and (39), we can write:

$$
\begin{aligned}
f\left(k^{\prime}(s+1)\right)-k^{\prime}(s+2) & =f\left(k^{\prime}(s+1)\right)-f(k(s+1))+f(k(s+1))-k(s+2) \\
& >\left[k^{\prime}(s+1)-k(s+1)\right]+f(k(s+1))-k(s+2) \\
& =[f(k(s))-c(s+2)-k(s+1)]+f(k(s+1))-k(s+2) \\
& =c(s+1)
\end{aligned}
$$

where the strict inequality in the second line of (40) follows from (2). This establishes (38). Using (36), (37) and (38), $\left\{k^{\prime}(t)\right\}$ is a feasible path from $\kappa$, with associated consumption sequence $\left\{c^{\prime}(t+1)\right\}$ given by $c^{\prime}(t+1)=f\left(k^{\prime}(t)\right)-k^{\prime}(t+1)$ for all $t \geq 0$. Further, (36) and (38) imply that $\{k(t)\}$ cannot be Suppes-Sen maximal, a contradiction. This establishes (33); clearly (32) and (33) establish (8), and $c(s+2)>c(s+1)$ for every $s \in\left\{t_{1}, t_{2}, \ldots\right\}$. As noted above, we also have $c(s+2)>f(z)-z$ for all $s \in\left\{t_{1}, t_{2}, \ldots\right\}$. Using (33), we see that for every $s \in\left\{t_{1}, t_{2}, \ldots\right\}$,

$$
c(s+1)=f(k(s))-k(s+1)<f(k(s))-k(s) \leq f(z)-z .
$$

This establishes (9) and hence part (ii) of the Proposition.

Proof of Lemma 6. We consider two cases separately: (i) $u\left(x, x^{\prime}\right)=0$; (ii) $u\left(x, x^{\prime}\right)>0$.

Case (i) In this case, we have:

$$
\begin{aligned}
u\left(x, x^{\prime}\right)+u\left(x^{\prime}, x^{\prime \prime}\right)+x^{\prime \prime}-x & =u\left(x^{\prime}, x^{\prime \prime}\right)+x^{\prime \prime}-x \leq f\left(x^{\prime}\right)-x^{\prime \prime}+x^{\prime \prime}-x \\
& =f\left(x^{\prime}\right)-x \leq f(f(x))-x \leq f(f(z))-z=\theta
\end{aligned}
$$

which establishes (10).

Case (ii) We subdivide this case as follows (a) $x \geq a$, (b) $x<a$. 
Case (ii)(a) In this subcase we have:

$$
\begin{aligned}
u\left(x, x^{\prime}\right)+u\left(x^{\prime}, x^{\prime \prime}\right)+x^{\prime \prime}-x & \leq f(x)-x^{\prime}+f\left(x^{\prime}\right)-x^{\prime \prime}+x^{\prime \prime}-x=[f(x)-x]+\left[f\left(x^{\prime}\right)-x^{\prime}\right] \\
& \leq[f(a)-a]+[f(z)-z]=f(f(z))-a+f(z)-z=f(f(z))-z=\theta
\end{aligned}
$$

which establishes (10).

Case (ii)(b) In this subcase, we have $x<a$, and

$$
f(x)-x^{\prime} \geq \theta
$$

by using (W). Then, using $x<a$, we have:

$$
f(x)<f(a)=f(f(z))
$$

So using (42) in (41),

$$
x^{\prime}<f(f(z))-\theta=z=1 .
$$

On the other hand, using (41),

$$
f(x) \geq x^{\prime}+\theta=x^{\prime}+f(a)-1 \geq f(a)-1=a+a b-b-1>a=f(z)
$$

the inequality in (44) following from the assumed condition (C). Then, (44) implies that:

$$
x>z=1 .
$$

The restrictions (43) and (44) are useful in evaluating the outputs from the stocks $x$ and $x^{\prime}$ precisely. Using (41) and (45),

$$
a+b(x-1)=f(x) \geq x^{\prime}+\theta=x^{\prime}+f(a)-1=x^{\prime}+a+b(a-1)-1
$$

so that $b x \geq x^{\prime}+(a b-1)$ and:

$$
x \geq\left[\frac{x^{\prime}}{b}+\frac{(a b-1)}{b}\right] \equiv m>1=z
$$

the strict inequality in (46) following from (C). Now, (46) implies that:

$$
f(x)-x \leq f(m)-m=a+b(m-1)-m=(a-b)-m(1-b) .
$$

Similarly using (43),

$$
f\left(x^{\prime}\right)-x^{\prime}=a x^{\prime}-x^{\prime}=(a-1) x^{\prime}
$$

Now, combining (47) and (48),

$$
\begin{aligned}
{[f(x)-x]+\left[f\left(x^{\prime}\right)-x^{\prime}\right] } & \leq(a-b)-m(1-b)+(a-1) x^{\prime} \\
& =(a-b)-\left[\frac{x^{\prime}}{b}+\frac{(a b-1)}{b}\right](1-b)+(a-1) x^{\prime} \\
& =(a-1) b+\frac{(1-b)}{b}+\left[(a-1)-\frac{(1-b)}{b}\right] x^{\prime} .
\end{aligned}
$$

Note that, using $(\mathrm{C}),(a-1) b=a b-b>1>(1-b)$, so that:

$$
\left[(a-1)-\frac{(1-b)}{b}\right]>0 \text {. }
$$


Using (43) and (50) in (49), we obtain:

$$
\begin{aligned}
{[f(x)-x]+\left[f\left(x^{\prime}\right)-x^{\prime}\right] } & \leq(a-1) b+\frac{(1-b)}{b}+\left[(a-1)-\frac{(1-b)}{b}\right] x^{\prime} \\
& \leq(a-1) b+\frac{(1-b)}{b}+\left[(a-1)-\frac{(1-b)}{b}\right]=(a-1)(b+1)=\theta .
\end{aligned}
$$

Using (51), we get:

$$
u\left(x, x^{\prime}\right)+u\left(x^{\prime}, x^{\prime \prime}\right)+x^{\prime \prime}-x \leq f(x)-x^{\prime}+f\left(x^{\prime}\right)-x^{\prime \prime}+x^{\prime \prime}-x=[f(x)-x]+\left[f\left(x^{\prime}\right)-x^{\prime}\right] \leq \theta
$$

which establishes (10) in case (ii)(b).

Proof of Lemma 7. Clearly $u(b, 1)=w(0)=0$, and $u(1, b)=w(a-b)=a-b \equiv \theta$. Thus, we have:

$$
\theta=u(b, 1)+u(1, b) .
$$

It remains to establish the inequality:

$$
u\left(x, x^{\prime}\right)+u\left(x^{\prime}, x^{\prime \prime}\right)+x^{\prime \prime}-x \leq \theta .
$$

We break up the demonstration into cases:(i) $x<b$ or $x^{\prime}<b$; (ii) $x \geq b$ and $x^{\prime} \geq b$. Clearly, we have:

$$
u\left(x, x^{\prime}\right)+u\left(x^{\prime}, x^{\prime \prime}\right)+x^{\prime \prime}-x \leq f(x)-x^{\prime}+f\left(x^{\prime}\right)-x^{\prime \prime}+x^{\prime \prime}-x=[f(x)-x]+\left[f\left(x^{\prime}\right)-x^{\prime}\right] .
$$

Thus, if $x<b$, then $f(x)-x<f(b)-b$ (since $b<z=1)=1-b$, while $f\left(x^{\prime}\right)-x^{\prime} \leq f(z)-z=a-1$, so that:

$$
[f(x)-x]+\left[f\left(x^{\prime}\right)-x^{\prime}\right]<(1-b)+(a-1)=(a-b) .
$$

Clearly, (54) also holds if $x^{\prime}<b$. Thus, (52) always holds in case (i).

We subdivide case (ii) into two subcases: (A) $f(x)-x^{\prime}<\theta$, (B) $f(x)-x^{\prime} \geq \theta$. In subcase (A), we have:

$$
\begin{aligned}
u\left(x, x^{\prime}\right)+u\left(x^{\prime}, x^{\prime \prime}\right)+x^{\prime \prime}-x & =u\left(x^{\prime}, x^{\prime \prime}\right)+x^{\prime \prime}-x \leq f\left(x^{\prime}\right)-x^{\prime \prime}+x^{\prime \prime}-x=f\left(x^{\prime}\right)-x \\
& \leq f(f(x))-x=f(f(x))-f(1)+f(1)-x \leq f_{+}^{\prime}(1)[f(x)-1]+a-x \\
& =b f(x)-x+(a-b) \leq b a x-x+(a-b)=a-b
\end{aligned}
$$

which establishes (52).

In subcase (B), we have $f(x) \geq(a-b)+x^{\prime} \geq(a-b)+b=a$, and so $x \geq 1$. Let $(x-1)=\varepsilon$; then $\varepsilon \geq 0$. Also, define $f(x)-x^{\prime}-\theta$ by $\delta$; then $\delta \geq 0$. Then $f(x)=f(1+\varepsilon)=a+b \varepsilon$, and so:

$$
\delta=f(1+\varepsilon)-x^{\prime}-\theta=a+b \varepsilon-x^{\prime}-(a-b)=b(1+\varepsilon)-x^{\prime} .
$$

This means $x^{\prime}=b(1+\varepsilon)-\delta$, and

$$
f\left(x^{\prime}\right)-x \leq a x^{\prime}-x=a b(1+\varepsilon)-a \delta-x=-a \delta .
$$

Using (56), we obtain:

$$
\begin{aligned}
u\left(x, x^{\prime}\right)+u\left(x^{\prime}, x^{\prime \prime}\right)+x^{\prime \prime}-x & \leq f(x)-x^{\prime}+f\left(x^{\prime}\right)-x^{\prime \prime}+x^{\prime \prime}-x \\
& =f(x)-x^{\prime}+f\left(x^{\prime}\right)-x=\theta+\delta-a \delta \leq \theta
\end{aligned}
$$

which again establishes (52). 
Proof of Lemma 8. Define $\beta=[f(b)-b]-[f(b-\alpha)-(b-\alpha)]$. Then $\beta>0$ since $f(k)-k$ is increasing in $k$ for $k \in[0,1), b \in(0,1)$ and $\alpha \in(0, b]$.

Suppose, contrary to (12), there is $\left(x, x^{\prime \prime}\right) \in \Lambda$, such that $L\left(x, x^{\prime \prime}\right)<\beta$. Then, $R\left(x, x^{\prime \prime}\right)>\theta-\beta$, and by definition of $R$, there is some $x^{\prime} \in \Gamma\left(x, x^{\prime \prime}\right)$, such that:

$$
u\left(x, x^{\prime}\right)+u\left(x^{\prime}, x^{\prime \prime}\right)+x^{\prime \prime}-x>\theta-\beta .
$$

However,

$$
\begin{aligned}
u\left(x, x^{\prime}\right)+u\left(x^{\prime}, x^{\prime \prime}\right)+x^{\prime \prime}-x & \leq f(x)-x^{\prime}+f\left(x^{\prime}\right)-x^{\prime \prime}+x^{\prime \prime}-x=f(x)-x+f\left(x^{\prime}\right)-x^{\prime} \\
& \leq f(x)-x+f(1)-1=f(x)-x+(a-1) \leq[f(b-\alpha)-(b-\alpha)]+(a-1) \\
& =[f(b)-b]-\beta+(a-1)=(1-b)-\beta+(a-1)=\theta-\beta
\end{aligned}
$$

the inequalities in the second line of (59) following from (2) and $0 \leq x \leq b-\alpha<b<1$. Clearly, (59) contradicts (58), establishing (12).

Proof of Theorem 1. (A) It is sufficient to prove (a), since then (b) follows from Proposition 1. Our proof of (a) is facilitated by considering three possibilities for the initial stock: (i) $\kappa \in[b, 1$ ); (ii) $\kappa \in[1, K]$; (iii) $\kappa \in[0, b)$.

Case (i) $(\kappa \in[b, 1)): \quad$ In this case, the consumption function $h$ generates the stock sequence $\{k(t)\}=$ $\{\kappa, f(\kappa), b, 1, b, 1, \ldots\}$ with associated consumption sequence $\{c(t+1)\}=\left\{0, f^{2}(\kappa)-b, 0, \theta, 0, \theta, \ldots\right\}$.

We verify that for each $t=0,2,4, \ldots$, the value $\operatorname{loss} L(k(t), k(t+2))=0$. For $t \in\{2,4, \ldots\}$, this follows from Lemma 7. For $t=0, L(k(t), k(t+2))=L(\kappa, b)$. Now, $f^{2}(\kappa)-b \geq f(1)-b=a-b$, so:

$$
\begin{aligned}
R(\kappa, b) & \geq u(\kappa, f(\kappa))+u(f(\kappa), b)+b-\kappa=u(f(\kappa), b)+b-\kappa=f^{2}(\kappa)-b+b-\kappa \\
& =f(a \kappa)-\kappa=a+b(a \kappa-1)-\kappa=a-b
\end{aligned}
$$

where the second line of (60) uses the facts that $\kappa \leq 1$ and $a \kappa \geq 1$ respectively. Thus, $L(\kappa, b) \leq 0$, and so by Lemma $7, L(\kappa, b)=0$.

We claim that $\{k(t)\}$ is w-maximal. If the claim is false, there is some feasible path $\left\{k^{\prime}(t)\right\}$ from $\kappa$, a positive number, $\alpha$, and a positive integer $N$, such that for all $T>N$,

$$
\alpha \leq \sum_{t=0}^{T}\left[u\left(k^{\prime}(t), k^{\prime}(t+1)\right)-u(k(t), k(t+1))\right] .
$$

For $T$ odd, write $T=2 S+1$, with $S$ a positive integer. Then:

$$
\begin{aligned}
\sum_{t=0}^{T} u\left(k^{\prime}(t), k^{\prime}(t+1)\right) & =\sum_{t=0}^{T} u\left(k^{\prime}(t), k^{\prime}(t+1)\right)+\sum_{s=0}^{S}\left[k^{\prime}(2 s+2)-k^{\prime}(2 s)\right]-\sum_{s=0}^{S}\left[k^{\prime}(2 s+2)-k^{\prime}(2 s)\right] \\
& =(S+1) \theta-\sum_{s=0}^{S} L\left(k^{\prime}(2 s), k^{\prime}(2 s+2)\right)-\left[k^{\prime}(T+1)-\kappa\right]
\end{aligned}
$$

and:

$$
\sum_{t=0}^{T} u(k(t), k(t+1))=(S+1) \theta-[k(T+1)-\kappa]
$$

since $L(k(2 s), k(2 s+2))=0$ for all $s \geq 0$. Combining (61), (62) and (63), one obtains:

$$
\alpha \leq\left[k(T+1)-k^{\prime}(T+1)\right]-\sum_{s=0}^{S} L\left(k^{\prime}(2 s), k^{\prime}(2 s+2)\right) .
$$


Since $k(T+1)=b$ for all $T$ odd, (64) implies that $k^{\prime}(T+1) \leq b-\alpha$ for all $T>N$, with $T$ odd. By Lemma 8 , we must then have for all $s$, satisfying $2 s>N+1$,

$$
\left.L\left(k^{\prime}(2 s), k^{\prime}(2 s+2)\right)\right) \geq \beta>0 .
$$

But, then, the right hand side of (64) becomes negative for large $T$, a contradiction.

Case (ii) $(\kappa \in[1, K]): \quad$ In this case, the consumption function $h$ generates the stock sequence $\{k(t)\}=$ $\{\kappa, b, 1, b, 1, \ldots\}$ with associated consumption sequence $\{c(t+1)\}=\{f(\kappa)-b, 0, \theta, 0, \theta, \ldots\}$. For each $t=$ $1,3,5, \ldots$, the value loss $L(k(t), k(t+2))=0$ by Lemma 7 .

We claim that $\{k(t)\}$ is w-maximal. If the claim is false, there is some feasible path $\left\{k^{\prime}(t)\right\}$ from $\kappa$, a positive number, $\alpha$, and a positive integer $N$, such that for all $T>N$,

$$
\alpha \leq \sum_{t=0}^{T}\left[u\left(k^{\prime}(t), k^{\prime}(t+1)\right)-u(k(t), k(t+1))\right] .
$$

For $T$ even, write $T=2 S$, with $S$ a positive integer. Then:

$$
\begin{aligned}
\sum_{t=1}^{T} u\left(k^{\prime}(t), k^{\prime}(t+1)\right) & =\sum_{t=1}^{T} u\left(k^{\prime}(t), k^{\prime}(t+1)\right)+\sum_{s=0}^{S-1}\left[k^{\prime}(2 s+3)-k^{\prime}(2 s+1)\right]-\sum_{s=0}^{S-1}\left[k^{\prime}(2 s+3)-k^{\prime}(2 s+1)\right] \\
& =S \theta-\sum_{s=0}^{S-1} L\left(k^{\prime}(2 s+1), k^{\prime}(2 s+3)\right)-\left[k^{\prime}(T+1)-k^{\prime}(1)\right]
\end{aligned}
$$

and:

$$
\sum_{t=1}^{T} u(k(t), k(t+1))=S \theta-[k(T+1)-k(1)]
$$

since $L(k(2 s+1), k(2 s+3))=0$ for $s \geq 0$. Also, since $f(\kappa)-b \geq f(1)-b=a-b$, we have $u(k(0), k(1))=$ $f(\kappa)-k(1)$, and:

$$
u\left(k^{\prime}(0), k^{\prime}(1)\right)-u(k(0), k(1)) \leq f(\kappa)-k^{\prime}(1)-f(\kappa)+k(1)=k(1)-k^{\prime}(1) .
$$

Combining (65), (66), (67), (68), one obtains:

$$
\alpha \leq \sum_{t=0}^{T}\left[u\left(k^{\prime}(t), k^{\prime}(t+1)\right)-u(k(t), k(t+1))\right] \leq k(T+1)-k^{\prime}(T+1)-\sum_{s=0}^{S-1} L\left(k^{\prime}(2 s+1), k^{\prime}(2 s+3)\right) .
$$

Since $k(T+1)=b$ for all $T$ even, (69) implies that $k^{\prime}(T+1) \leq b-\alpha$ for all $T>N$, with $T$ even. By Lemma 8 , we must then have for all $s$, satisfying $2 s>N$,

$$
\left.L\left(k^{\prime}(2 s+1), k^{\prime}(2 s+3)\right)\right) \geq \beta>0 .
$$

But, then, the right hand side of (69) becomes negative for large $T$, a contradiction.

Case (iii) $(\kappa \in[0, b)): \quad$ Since $f(0)=0$, the case in which $\kappa=0$ is trivial. Thus, we focus on the case in which $\kappa \in(0, b)$. Define the sequence $\{K(t)\}$ by $K(0)=\kappa$, and $K(t+1)=f(K(t))$ for $t \geq 0$. Then $\{K(t)\}$ is an increasing sequence, which converges to $K$. Consequently, we can find the smallest time period, $\tau$, such that $K(\tau) \geq b$; clearly, $\tau \geq 1$. The consumption function $h$ generates the stock sequence $\{k(t)\}=\{\kappa, K(1), \ldots, K(\tau), K(\tau+1), b, 1, b, 1, \ldots\}$ with associated consumption sequence $\{c(t+$ $1)\}=\{0, \ldots, 0, f(K(\tau+1))-b, 0, \theta, 0, \theta, \ldots\}$.

We claim that $\{k(t)\}$ is w-maximal. If the claim is false, there is some feasible path $\left\{k^{\prime}(t)\right\}$ from $\kappa$, a positive number, $\alpha$, and a positive integer $N$, such that for all $T>N$,

$$
\alpha \leq \sum_{t=0}^{T}\left[u\left(k^{\prime}(t), k^{\prime}(t+1)\right)-u(k(t), k(t+1))\right]
$$


Consider the $T$, which can be written as $T=2 S+\tau+1$, with $S$ a positive integer. Then:

$$
\begin{aligned}
\sum_{t=\tau}^{T} u\left(k^{\prime}(t), k^{\prime}(t+1)\right) & =\sum_{t=\tau}^{T} u\left(k^{\prime}(t), k^{\prime}(t+1)\right) \\
& +\sum_{s=0}^{S}\left[k^{\prime}(2 s+\tau+2)-k^{\prime}(2 s+\tau)\right]-\sum_{s=0}^{S}\left[k^{\prime}(2 s+\tau+2)-k^{\prime}(2 s+\tau)\right] \\
& =(S+1) \theta-\sum_{s=0}^{S} L\left(k^{\prime}(2 s+\tau), k^{\prime}(2 s+\tau+2)\right)-\left[k^{\prime}(T+1)-k^{\prime}(\tau)\right]
\end{aligned}
$$

and:

$$
\sum_{t=\tau}^{T} u(k(t), k(t+1))=(S+1) \theta-[k(T+1)-k(\tau)]
$$

since $L(k(2 s+\tau), k(2 s+\tau+2))=0$ for all $s \geq 0$ (for the reason given in Case (i) above). Combining (71) and (72), one obtains:

$$
\begin{aligned}
& \sum_{t=\tau}^{T} u\left(k^{\prime}(t), k^{\prime}(t+1)\right)-\sum_{t=\tau}^{T} u(k(t), k(t+1)) \\
& =\left[k^{\prime}(\tau)-k(\tau)\right]+\left[k(T+1)-k^{\prime}(T+1)\right]-\sum_{s=0}^{S} L\left(k^{\prime}(2 s+\tau), k^{\prime}(2 s+\tau+2)\right) .
\end{aligned}
$$

We now show that:

$$
\left[\sum_{t=0}^{\tau-1} u\left(k^{\prime}(t), k^{\prime}(t+1)\right)-\sum_{t=0}^{\tau-1} u(k(t), k(t+1))\right] \leq\left[k(\tau)-k^{\prime}(\tau)\right] .
$$

Note that $k(t+1)=f(k(t))$ for $t=0, \ldots, \tau-1$, and so:

$$
\sum_{t=0}^{\tau-1} u(k(t), k(t+1))=0
$$

Note that $k^{\prime}(t) \leq k(t)<b$ for $t=0, \ldots, \tau-1$, so $\left[f\left(k^{\prime}(t)\right)-k^{\prime}(t)\right] \leq[f(k(t))-k(t)]$ for $t=0, \ldots, \tau-1$. Consequently,

$$
\begin{aligned}
\sum_{t=0}^{\tau-1} u\left(k^{\prime}(t), k^{\prime}(t+1)\right) & \leq \sum_{t=0}^{\tau-1}\left[f\left(k^{\prime}(t)\right)-k^{\prime}(t+1)\right]=\sum_{t=0}^{\tau-1}\left[f\left(k^{\prime}(t)\right)-k^{\prime}(t)\right]+\left[\kappa-k^{\prime}(\tau)\right] \\
& \leq \sum_{t=0}^{\tau-1}[f(k(t))-k(t)]+\left[\kappa-k^{\prime}(\tau)\right] \\
& =\sum_{t=0}^{\tau-1}[f(k(t))-k(t+1)]-[\kappa-k(\tau)]+\left[\kappa-k^{\prime}(\tau)\right]=\left[k(\tau)-k^{\prime}(\tau)\right]
\end{aligned}
$$

Clearly, (74) follows from (75) and (76).

Combining (70), (73) and (74), we obtain:

$$
\begin{aligned}
\alpha & \leq \sum_{t=0}^{T}\left[u\left(k^{\prime}(t), k^{\prime}(t+1)\right)-u(k(t), k(t+1))\right] \\
& \leq\left[k(T+1)-k^{\prime}(T+1)\right]-\sum_{s=0}^{S} L\left(k^{\prime}(2 s+\tau), k^{\prime}(2 s+\tau+2)\right) .
\end{aligned}
$$


Since $k(T+1)=b$ for all $T$ of the form $T=2 S+\tau+1$, (77) implies that $k^{\prime}(T+1) \leq b-\alpha$ for all $T>N$, with $T$ of the form $T=2 S+\tau+1$. By Lemma 8, we must then have for all $s$, satisfying $2 s+\tau+2>N+1$,

$$
\left.L\left(k^{\prime}(2 s+\tau+2), k^{\prime}(2 s+\tau+4)\right)\right) \geq \beta>0 .
$$

But, then, the right hand side of (77) becomes negative for large $T$, a contradiction.

This completes the proof of (a), and therefore of (A). The proof of (B) is straightforward, since the dynamical system is given by (DS).

\section{References}

[1] Asheim, G.B., W. Bossert, Y. Sprumont and K. Suzumura, Infinite-Horizon Choice Functions, Economic Theory 43 (2010) 1-21.

[2] Asheim, G.B., W. Buchholz and B. Tungodden, Justifying Sustainability, J. Environmental Economics and Management 41 (2001), 252-268.

[3] d'Aspremont, C., Axioms for Social Welfare Orderings, in L. Hurwicz, D. Schmeidler and H. Sonnenschein (eds.), Social Goals and Social Organizations: Essays in Memory of Elisha Pazner, Cambridge University Press, Cambridge, (1985), 19-76.

[4] Atsumi, H., Neoclassical growth and the efficient program of capital accumulation, Rev. Econ. Stud. 32 (1965) 127-136.

[5] Basu, K. and T. Mitra, Utilitarianism for infinite utility streams: A new welfare criterion and its axiomatic characterization, J. Econ. Theory 133 (2007) 350-373.

[6] Brock, W.A., On existence of weakly maximal programmes in a multi-sector economy, Rev. Econ. Stud. 37 (1970) 275-280.

[7] Cass, D., On Capital Overaccumulation in the Aggregative, Neoclassical Model of Economic Growth, J. Econ. Theory 4 (1972) 200-223.

[8] Gale, D., On Optimal Development in a Multi-Sector Economy, Rev. Econ. Stud. 34 (1967), 1-18.

[9] Hardy, G.H., J.E. Littlewood and G. Polya, Inequalities, Second Edition, Cambridge University Press, Cambridge, 1952.

[10] Koopmans, T.C., Intertemporal Distribution and Optimal Aggregate Economic Growth, in William Fellner et al, Ten Economic Studies in the Tradition of Irving Fisher, Wiley, New York, (1967), 95126.

[11] McKenzie, L.W., Accumulation programs of maximum utility and the von Neumann facet, Value, Capital and Growth, ed. J.N. Wolfe, Edinburgh University Press, (1968), 353-383.

[12] Radner, R., Paths of economic growth that are optimal with regard only to final states: A turnpike theorem, Rev. Econ. Stud. 28 (1961), 98-104.

[13] Ramsey, F., A Mathematical Theory of Saving, Economic Journal 38 (1928), 543-559.

[14] Sen, A.K., Collective Choice and Social Welfare; Edinburgh, Oliver \& Boyd, 1971.

[15] Suppes, P., Some Formal Models of Grading Principles, Synthese 6 (1966), 284-306.

[16] Svensson, L.-G., Equity among Generations, Econometrica 48 (1980), 1251-1256. 